\title{
Diaspora, migration, and the sciences: a new integrated perspective
}

\author{
Leonard Rutgers ${ }^{1} \cdot$ Harry Ostrer $\mathbb{1}^{2} \cdot$ Tracy Prowse $\mathbb{1}^{3} \cdot$ Hannes Schroeder ${ }^{4}$
}

Received: 12 November 2018 / Accepted: 27 November 2018 / Published online: 19 December 2018

(c) European Society of Human Genetics 2018

The study of human migration since the Bronze Age is ready for a new view as archeological studies incorporate ancient DNA (aDNA) analysis of human, animal and plant remains, pathogens and the systematic use of big data. Prior archeology revolutions occurred with the advent of radiocarbon dating (1950s) and stable isotope analysis (1980s) and contributed to the understanding of who moved and when [1]. Using these methods, archeologists and historians sought to understand whether the few or the many contribute their ideas, genes or both to the creation of new communities, languages and material cultures or the transformation of existing ones. Future insights will be derived not only from the studies of texts, funerary inscriptions and practices, grave goods, settlement patterns, and stable isotope analysis of human, faunal and floral remains, but also from aDNA. On 3-7 September 2018, historians, archeologists, physical anthropologists, and geneticists subjected these methods to scrutiny at a conference at the Lorentz Center at Leiden University. Though their presentations and discussions, they agreed that a consilience view of human migration might be derived from comparative analysis [2]. Several takeaways were provided that can inform the genetics community.

\section{Archaeological and genetic studies need to be performed in tandem to test hypotheses about migration}

Sometimes groups can be identified through their specific material culture or, if inscriptions are available, through the

\footnotetext{
$\triangle$ Harry Ostrer

harry.ostrer@einstein.yu.edu

1 Utrecht University, Utrecht, Netherlands

2 Albert Einstein College of Medicine, Bronx, NY, USA

3 McMaster University, Hamilton, ON, Canada

4 Natural History Museum of Denmark, Copenhagen, Denmark
}

self-identity that emerges from such texts, which sometimes includes references to place of origin [3]. Groups can also be identified by cluster analysis of biological data (e.g., based on single nucleotide variants (SNVs) and identicalby-descent (IBD) segments) and strontium and oxygen isotope data can provide insights on lifetime mobility [4]. Statistical methods that have been applied to genetic data to define contemporary groups (e.g., genetic distances, principal component analysis ADMIXTURE analysis, and three-population and four-population tests) are also applicable to ancient groups. However, results have to be interpreted with caution. Outliers may be representative of migrants (a scenario that can, in turn, be tested by isotopic analyses), hybrid ancestry or a broader representation of group membership than was previously characterized. Outliers within isotopic group analysis are commonly thought to represent migrants, although the place of migration may be difficult to establish in the absence of isotopic maps relating to the pertinent timeframes [4].

\section{Events can be timed, especially population bottlenecks, time to a most recent common ancestor and admixture}

The timing is more accurate when IBD segmental analytical methods are applied than when de novo mutational analysis is applied, because of inconstancy of the molecular clock. IBD analyses, including total sharing and distribution of segmental lengths across groups, identify shared ancestry with longer shared segments indicating more recent ancestry. Greater segmental sharing and runs of homozygosity within a group indicate consanguinity. High fractional segmental sharing between individuals is indicative of kinship [5]. Unsupervised clustering of shared segment assigns individual to groups whose ancestral histories may be known or may suggest new histories that could be tested using other complementary methods [6]. Segmental sharing across groups and across geographical locales provides evidence for migration and admixture for Diaspora and 
migratory groups [5]. All of this should be accompanied by a program of rigorous radiocarbon dating.

\section{Study design, project scale, and sampling strategy matter}

The study design must relate to the history of the subjects tested, if meaningful inferences are to be made. The study should be conducted in compliance with national and local laws and with sensitivity to community practices, acknowledging potential ancestry to contemporary communities [7]. Because aDNA studies are commonly performed on skeletal remains, the provenancing should be informed by burial inscriptions, grave goods and funerary practices that indicate group membership. Although single genomes can yield significant insights about individuals and even populations [8], most studies would benefit from an adequate sample size, wherein specific hypotheses have been formulated and prior power calculation have been performed to test sufficiency of sample size. For some archeological sites, broad sampling may provide a coarse grain view of the genetic makeup of the site, especially when the site has been used over hundreds of years. Intensive sampling of multiple individuals in the same grave or in contiguous graves can address issues of kinship that would otherwise be missed. In situations where different cultural groups are known to have existed alongside one another, analysis should extend to all pertinent groups [9]. All studies should adhere to best possible standards of aDNA data generation, including DNA extraction, library preparation, and sequencing. If necessary, targeted enrichment techniques should be employed [10]. Sequencing across the genome should be performed to enable SNV as well as IBD analysis of autosomes and $\mathrm{X}$ chromosomes and haplogroup analysis of $\mathrm{Y}$ chromosome and mitochondrial DNA. Given the limited availability of aDNA, resampling and resequencing may be impossible, so best effort must be mounted upfront. Whenever possible, samples should be archived in anticipation of future technology improvements.

The past and the present inform each other; thus, large-scale datasets should be made readily accessible to the research community through public repositories, such as the European Nucleotide Archive. Ancient genome data can provide frames of reference for modern populations. Populations that were thought to have vanished (e.g., the Caribbean Taino) can be observed in the genomes of contemporary groups and, thus, provide insight about stability, migration, and admixture. This puts responsibility on researchers to ensure that new data and research findings are published and presented in ways that are informative and sensitive to descendant communities.

\section{Compliance with ethical standards}

Conflict of interest The authors declare that they have no conflict of interest.

\section{References}

1. Kristiansen K. Towards a New Paradigm? The Third Science Revolution and its Possible Consequences in Archaeology. Curr Swed Archeol. 2014;22:11-34.

2. Johannsen JJ, Larson G, Meltzer DD, Vander Linden M. A composite window into human history. Science. 2017;356:1118 20.

3. Nielsen R, Akey JM, Jakobsson M, Pritchard JK, Tishkoff S, Willerslev E. Tracing the peopling of the world through genomics. Nature. 2017;541:302-10.

4. Laffoon JE, Sonnemann TF, Shafie T, Hofman CL, Brandes U, Davies GR. Investigating human geographic origins using dualisotope $(87 \mathrm{Sr} / 86 \mathrm{Sr}$, elta18O) assignment approaches. PLoS ONE. 2017; 12:e172562.

5. Hellenthal G, Busby GBJ, Band G, Wilson JF, Capelli C, Falush D, et al. A genetic atlas of human admixture history. Science. 2014;343:747-51.

6. Han E, Carbonetto P, Curtis RE, et al. Clustering of 770,000 genomes reveals post-colonial population structure of North America. Nat Commun. 2017;8:14238.

7. Bardill J, Bader AC, Garrison NA, et al. Advancing the ethics of paleogenomics. Science. 2018;360:384-5.

8. Schroeder H, Sikora M, Gopalakrishnan S, et al. Origins and genetic legacies of the Caribbean Taino. Proc Natl Acad Sci USA. 2018;115:2341-6.

9. O'Sullivan N, Posth C, Coia V, et al. Ancient genome-wide analyses infer kinship structure in an Early Medieval Alemannic graveyard. Sci Adv. 2018;4:eaao1262.

10. Mathieson I, Lazaridis I, Rohland N, et al. Genome-wide patterns of selection in 230 ancient Eurasians. Nature. 2015;528:499-503. 